\title{
Development of the Conformity to Feminine Norms Inventory
}

\author{
James R. Mahalik, ${ }^{1,3}$ Elisabeth B. Morray, ${ }^{1}$ Aimée Coonerty-Femiano, ${ }^{1}$ \\ Larry H. Ludlow, ${ }^{1}$ Suzanne M. Slattery, ${ }^{1}$ and Andrew Smiler ${ }^{2}$
}

\begin{abstract}
This article describes the construction of the Conformity to Feminine Norms Inventory (CFNI), which was designed to assess women's conformity to an array of feminine norms found in the dominant culture in the United States. In addition, we present four studies in which the psychometric properties of the CFNI were examined. In Study 1, factor analysis indicated that the CFNI is comprised of eight distinct factors labeled as Nice in Relationships, Thinness, Modesty, Domestic, Care for Children, Romantic Relationship, Sexual Fidelity, and Invest in Appearance. Results from Study 2 indicated that the CFNI has strong internal consistency estimates and differentiates college women from college men. In addition, Study 2 demonstrated that the CFNI Total score and subscale scores relate to Bem Sex Role Inventory and Feminist Identity Development Scale scores in theoretically consistent patterns. Study 3 indicated that the CFNI Total score and several of the subscales significantly and positively relate to scores on the Eating Disorder Inventory. Finally, Study 4 demonstrated that the CFNI Total score and subscale scores have high test-retest estimates for a 2-3 week period. The discussion focuses on potential uses of the CFNI, limitations to the study, and suggestions for future research.
\end{abstract}

KEY WORDS: femininity; gender roles; women; feminine norms.

Gender role norms share the characteristics of social norms, which are described as "rules and standards that are understood by members of a group, that guide and/or constrain social behavior without the force of laws" (Cialdini \& Trost, 1999, p. 152). For example, social agents such as parents, teachers, peers, and the media teach women and men the rules and standards of femininity and masculinity, respectively (Bem, 1981a; Lytton \& Romney, 1991; Meth, 1990). Research confirms that this learning occurs very early in life, as evidenced by findings that by age 5 children have already developed clearly defined notions of what constitutes appropriate behavior for men and women (see Lytton \& Romney, 1991,

\footnotetext{
${ }^{1}$ Boston College, Chestnut Hill, Massachusetts.

${ }^{2}$ University of New Hampshire, Durham, New Hampshire.

${ }^{3}$ To whom correspondence should be addressed at Campion Hall 312, Boston College, Chestnut Hill, Massachusetts 02467; e-mail: Mahalik@bc.edu.
}

for a review). Gender role norms also provide guidance for women and men about how they are supposed to act, think, and feel, as well as constrain women and men from certain behaviors that are "off limits" (Gilbert \& Scher, 1999). Like other social norms, gender role norms are typically maintained by the reinforcement or punishment of social responses (Locksley \& Colten, 1979).

We also know that gender role norms are important in the lives of women and men in that they foster identity development (e.g., Bem, 1981a; Chodorow, 1978; Kagan, 1964; Kohlberg, 1966), can contribute to gender role strain (Eisler, 1995; Pleck, 1981, 1995), and are viewed by many as important to integrate into the process of counseling and psychotherapy (Brown, 1986; Gilbert \& Scher, 1999; Brooks \& Good, 2001). As such, mental health practitioners increasingly view gender roles as critical to their theoretical, empirical, and clinical work. 
We view the current state of femininity measures as inadequate to address these purposes because, although a large number of normative messages have been identified by scholars as associated with femininity (e.g., be nice, focus on relationships, be attractive, be thin, nurture others, be silent, defer to men, and be domestic, just to name a few; see Crawford \& Unger, 2000; Gilbert \& Scher, 1999), most current measures examine femininity only as a global construct. For example, the most widely used measure of gender roles, the Bem Sex Role Inventory (Bem, 1981b), assesses femininity as a single score made up of responses to stereotypical and socially desirable feminine characteristics, which results in a global indicator of the extent to which the inventory taker is "feminine."

Some have criticized this measure because they view it as assessing instrumental and expressive personality traits rather than gender roles per se, and concluded that it does not actually measure femininity or masculinity (Betz, 1995; Spence \& Helmreich, 1980). Our central criticism of the Bem Sex Role Inventory is that it, and other measures that assess femininity in a global way (e.g., MMPI-2 Feminine Gender Role Scale), are unable to differentiate among the many distinct cultural injunctions that define the social construction of femininity in U.S. society today.

We believe that Tolman and Porche's (2000) Adolescent Femininity Ideology Scale is a step in the right direction as it focuses on the identification of specific feminine injunctions that adolescent girls experience. However, we see that measure as limited in that it only identifies two feminine ideologies (i.e., Inauthentic Self in Relationship and Objectified Relationship with Body) and was developed for use by girls and adolescents, not adult women.

We make this criticism about global measures of femininity because we take a normative perspective that views gender roles as comprised of a large number of culturally based ideologies that script gender relations, attitudes, and beliefs (see Thompson \& Pleck, 1995). From this perspective, women in the U.S. are understood as experiencing many different sociocultural injunctions that define traditional feminine roles (to nurture children, to be physically attractive, to be submissive, and so on). Any inventory that purports to assess femininity should also assess the salience of the different feminine norms for individual women. Such a measure would be able to incorporate the fact that one woman may construct the most salient features of her femininity as car- ing for children and being domestic, whereas another woman may construct the most salient features of her femininity as being thin and physically attractive. This critical variability gets lost in any global index of femininity. Femininity inventories must address this variability in order for researchers both to understand the effects that specific feminine norms have on individual women and to explore the contextual influences that give rise to each woman's constructions of femininity.

The purpose of this study was to develop a psychometrically sound measure that allows researchers and clinicians to assess women's degree of conformity to an array of feminine norms in the dominant culture in the U.S. (i.e., White, middle and upper-class, heterosexual). Conformity to feminine norms is defined as adhering to societal rules and standards about how to be feminine and is demonstrated in the individual woman's behaviors, feelings, and thoughts. For example, conformity would be reflected in actions consistent with the feminine norm, positive feelings (e.g., pride) related to conformity, and negative feelings (e.g., shame) related to nonconformity, and having beliefs that emphasize the importance of the norm. Conversely, nonconformity to feminine norms is defined as not adhering to societal expectations for what constitutes femininity.

Feminine norms from the dominant culture in the U.S. were chosen because feminine ideologies from the dominant European American and middleclass culture in the U.S. are thought to be the most pervasive and powerful in U.S. society (Brown, 1998; Mahalik et al., 2003). We believe that the expectations of femininity as constructed by European American, middle and upper class heterosexuals in the U.S. should affect members of that group, as well as every other woman in U.S. society who is held up to those standards and who experiences acceptance or rejection from the majority culture, in part, based on adherence to the powerful group's femininity norms.

In this article, a factor analysis and three other studies of the reliability and validity of the Conformity to Feminine Norms Inventory (CFNI) are presented. Specifically, in Study 1, the factor structure of the proposed inventory was examined. In Study 2, the internal consistency estimates of the CFNI were examined, and data from both women and men were used to examine the discriminative validity. In addition, we were interested in testing the convergent and concurrent validity of the measure. To do so, we examined the CFNI in relation to femininity, 
masculinity, and feminist identity (Study 2), as well as its relatedness to eating disorder and body image issues (Study 3). In Study 4, we examined additional evidence for the CFNI's reliability by analyzing the stability of CFNI scores over a 2-3 week time period.

\section{Construction of the Conformity to Femininity Norms Inventory (CFNI)}

The first step in constructing the measure was to identify feminine norms in the dominant culture in the U.S. This consisted of several steps; we started by reviewing the literature on traditional feminine norms in the U.S. (e.g., Carpenter, 2002; Crawford \& Unger, 2000; Deaux \& Lewis, 1984; Deutsch \& Saxon, 1998; Eagly \& Steffen, 1984; Gilbert \& Scher, 1999; Gillespie, 2003; Gillespie \& Eisler, 1992; Gilligan, 1982; Hurd, 1999; Jordon, 1992; Matschiner \& Murnen, 1999; Morawski, 1985; Rudman \& Glick, 2001; Spence \& Helmreich, 1978).

Next, 32 women participated in five focus groups that consisted of 4-9 members each. The women averaged 32.53 years of age $(S D=10.09)$ and were predominantly European American (30 European American, 1 Hispanic/Latina, and 1 biracial). Most women reported that they were single (19 single, 4 same sex partnership, 4 married, 3 divorced, and 2 living together with cross or other sex partner) and heterosexual (22 heterosexual, 6 lesbian, 4 bisexual). They were recruited from a college campus in the northeast and through contacts of the researchers. These women met with one of two female researchers to identify feminine norms. One of the focus groups was comprised of undergraduate women, two of the groups were comprised of masters students in a counseling psychology program, and two of the groups were comprised of adult women in the community. They met on average for $90 \mathrm{~min}$ and were asked to "Reflect on your experiences as women and identify messages that you hear being given to women in the United States about how women are supposed to act, think, and feel, as well as what benefits and costs women experience when they either endorse or reject these messages."

Following the group interviews, two of the authors listened to the tapes of the focus groups and recorded the messages that the women in the group identified (e.g., women are expected to establish and maintain relationships, women should stay at home and run the household, women should try to look attractive). This list was added to the list identified from the literature review about traditional feminine norms, and the result was more than 100 separate messages identified through the literature review and focus groups. Next, because many of the messages identified seemed to share similarities, two of the researchers placed these statements (e.g., "women are expected to establish and maintain relationships") on $3 \times 5$ cards and sorted them into clusters. After grouping the messages into 13 large clusters, the two researchers read the cards in each cluster, and then each cluster was labeled. This resulted in the following feminine norms being identified: "Self as Mother," "Be Relational-Connected," "Be Silent," "Be Dependent," "Be Married," "Look Young," "Thinness," "Be Physically Attractive-Ornamental," "Rely on Men," "Be Pleasant," "Defer to Men," "Be Virginal," and "Be Sexy." Because several items were not easily clustered (e.g., "women are expected to be blonde and blue-eyed") or were so global as to fit under several more specific clusters (e.g., "women are expected to be heterosexual" underlies the imperatives to be married, rely on men, be virginal, be sexy), we eliminated these types of items from further consideration.

Finally, two focus groups ( $n \mathrm{~s}=6$ and 7 ) that consisted of both women and men who were masters and doctoral students in counseling psychology met with the senior author. These groups met every week for 90 min over an 8-month period to (a) discuss examples for the norms identified, (b) refine the categories of the norms, and (c) construct items to assess the continuum of conformity to the norm.

Through discussion of the 13 categories, and through attempts to write items for each of the norms, the categories were further refined into 12 feminine norms that were incorporated into the survey: Relational, Sweet and Nice, Thinness, Put Others First, Look Young, Sexy, Modesty, Domestic, Caring for Children, Romantic Relationship, Sexual Fidelity, and Invest in Appearance. When we constructed the items, our goal was to reflect the full continuum of conformity that each feminine norm could have, ranging from extreme conformity, to moderate conformity, to moderate nonconformity, and finally to extreme nonconformity. For example, for the norm of Relational, the item "It would be awful if I ever lost touch with someone in my life" was written as an item that reflects extreme conformity to the Relational norm. "I feel good about myself when others know that I care" reflects moderate conformity to the Relational norm. "I don't feel guilty if I lose contact with a friend" reflects moderate nonconformity 
to the Relational norm, and "I would feel burdened if I had to maintain relationships with others" reflects extreme nonconformity to the Relational norm.

The focus groups wrote items to incorporate affective (i.e., feelings about conforming or not conforming to that norm), behavioral (i.e., actions that reflect either conformity or nonconformity to that norm), and cognitive aspects (i.e., self-talk or beliefs about the importance of conformity on nonconformity to that norm) for each of the four degrees of conformity. Following this format, the focus groups constructed 12 items for each of the identified norms (i.e., one affective, one behavioral, and one cognitive item for each of the four of conformity statuses). In this way, 144 initial items were constructed such that all items assessed either an affective, behavioral, or cognitive dimension for each of the four conformity statuses for all 12 femininity norms. For example, the item "I have a long, methodical daily beauty routine" was constructed to assess extreme conformity behaviors that reflect the feminine norm of Invest in Appearance.

The measure was then piloted at four different times with small groups of college-aged female and male undergraduate participants ( $n$ s ranged from 20 to 30) both to solicit their reactions to the items and to assess the subscales' internal consistency estimates. Using these pilot data, the focus groups discussed both the reactions from participants and the results from the internal consistency analyses. We also examined this data to determine if extreme and moderate items performed as we expected. Because agreeing with extreme conformity and extreme nonconformity items should occur less frequently on average, mean scores from the pilot participants were examined to determine if extreme items were being endorsed at lower rates than moderate items. When moderate items and extreme items had similar scores, they were both edited to reflect either greater rigidity (i.e., extreme conformity or nonconformity) or greater flexibility (i.e., moderate conformity or nonconformity). Based on the input and responses to the items from the pilot groups, we revised items to improve the readability of items, the internal consistency estimates of the 12 subscales, and the validity of the extreme and moderate items.

Through this process, the Conformity to Feminine Norms Inventory was developed. Respondents to the CFNI indicate how much they agreed with the initial 144 items using a 4-point scale where $S D=$ Strongly Disagree, $D=$ Disagree, $A=$ Agree, and $S A=$ Strongly Agree. This 4-point scaling was adopted to be consistent with the structure of the theoretical continuum of conformity that Mahalik et al. (2003) posited in which conformity to a specific gender role norm could range across the four conformity statuses (i.e., extreme conformity, moderate conformity, moderate nonconformity, and extreme nonconformity).

\section{STUDY 1}

To identify the factor structure of the CFNI, and inform us as to how the 144-item inventory might be revised, a series of factor analyses were performed on the full $144 \times 144$ item correlation matrix. Analyses were performed with the SPSS FACTOR procedure (SPSS, 1999).

\section{Method}

\section{Participants and Procedure}

Seven hundred and thirty-three women participated in the study. They averaged 19.72 years of age $(S D=2.69)$, and most of them were European American (592 European American, 21 African American, 59 Asian or Asian American, 34 Hispanic/Latina, 2 Native American, 16 biracial, and 9 described themselves as "Other"). Most women reported that they were single (667 single, 7 married, 1 divorced, 1 in committed same-sex relationship) and heterosexual ( 714 heterosexual, 6 lesbian, and 13 bisexual). These women were recruited from college campuses in the northeast and through both classroom recruitment and psychology human subjects pools to complete the CFNI and other instruments described in Studies 2 and 3.

\section{Results}

Prior to conducting the factor analyses, three tests were performed to ensure that the correlation matrix had variation suitable for factoring. First, we verified that the determinant was nonzero, that is, that no perfect linear dependencies existed among the items (Green, 1976). Second, the KaiserMeyer-Olkin measure of sampling adequacy was .86, described by Kaiser (1974) as "meritorious," which supports the strength of the overall factorial determination. Third, Bartlett's test of sphericity (Bartlett, 
1950) was statistically significant $(p<.001)$, which indicates that the population correlation matrix is not an identity matrix.

A principal axis extraction technique was employed because after each factor was extracted this technique accounts for the greatest variance that remains in the residual correlation matrix. Oblique rotations (Oblimin) were performed because of the presumed correlated nature of the factors, and item factor loadings below 3 were not considered. The criteria for deciding on the final factor solution included the magnitude of the eigenvalues, the percent of variance accounted for by each factor, the overall percent of variance accounted for, the number of items that loaded highly on each factor, and the interpretability of the factors after the items that loaded most highly on that factor were examined.

An exploratory factor approach was used to identify the structure of the CFNI. The initial criterion of a minimum eigenvalue greater than 1.00 produced 34 factors, which clearly was not a parsimonious factor solution. Because the inventory was developed to assess 12 distinct femininity norms, a series of step-down factor analyses were conducted beginning with 12 factors. Oblique rotations were performed using the Oblimin criterion in SPSS, and each of the solutions were examined for interpretability. Following this procedure, a 10 -factor solution appeared to be the strongest in terms of clear interpretability of the 144 items.

At this point, however, we thought that the 10factor solution with 144 items was still not satisfactory, as a number of items did not contribute to these 10 factors. For example, the items that comprised the Look Young scale did not load exclusively on any factor and had extremely low communality values in the range of .0-.2. Thus, to remove error associated with these items and reduce the length of the CFNI, the Look Young items were removed, and factor analyses were re-run. Subsequent to these analyses, Put Others First and Sexy items were also removed, as they were dispersed across several scales and did not define a unique structure.

Ultimately, the final solution consisted of 118 items that defined eight subscales that accounted for $39.51 \%$ of the variance. It is noteworthy that a similar 8 -factor solution with the 144 items only accounted for $34.6 \%$ of the variance. Thus, our removal of the 36 items from the factor analysis resulted in less redundancy and error variance in our solution.

The results of this final 8-factor, principal axis, oblique rotated solution are reported in Table I. The table contains the factor loadings, communality loadings, eigenvalues, and percent of variance statistics.

We next examined each of the 118 items to determine on which of the eight factors each item significantly and most strongly loaded, which items had the highest loadings on each factor, and whether those high loading items on each factor were identifiable as representing a single construct (see Table I). Items were retained for the subscales only if they loaded at $|.4|$ or higher on one factor and did not cross-load any higher than $|.3|$ on any other factor. Use of these retention criteria resulted in 84 items being retained, with 7 of the 8 factors comprised of items exclusively from only one of the original rationally derived subscales. For example, all items that met the retention criteria for Factor 2 were developed initially to reflect the norm of being thin. Therefore, this factor was labeled Thinness. Using this rationale, Factors 2 through 8 were labeled as Thinness, Modesty, Domestic, Care for Children, Romantic Relationship, Sexual Fidelity, and Invest in Appearance. Factor 1 was composed of items initially intended to measure two feminine norms (i.e., investing in relationships and being sweet and nice). Thus, in examining the items that comprised Factor 1, we concluded that Factor 1 should be labeled as Nice in Relationships.

Next, we examined the magnitude of the factor correlations to determine the amount of overlap between factors. Results indicated that only 2 of the 28 pairs of correlations among the eight factors were equal to, or larger than |.3| [i.e., a correlation of .38 between Factor 1 (Nice in Relationships) and Factor 5 (Care for Children), and a correlation of .30 between Factor 2 (Thinness) and Factor 8 (Invest in Appearance)]. However, apart from these two relationships, the low factor correlations suggest that the factors identified from this sample were relatively distinct from each other.

\section{Discussion}

Results from the factor analysis supported our view that the femininity norms we identified and quantified through the measure development process were distinct from each other. Our results suggest that the CFNI is an 84-item inventory with an 8-factor structure whose factors are best labeled as Nice in Relationships, Thinness, Modesty, Domestic, Care for Children, Romantic Relationship, Sexual Fidelity, and Invest in Appearance. The retained items in each dimension reflect behaviors, 
Table I. Principal Axis Oblimin Rotated Factor Matrix, Communality, Eigenvalues, and Percentage of Total Variance for the Eight Conformity to Feminine Norms Inventory Factors

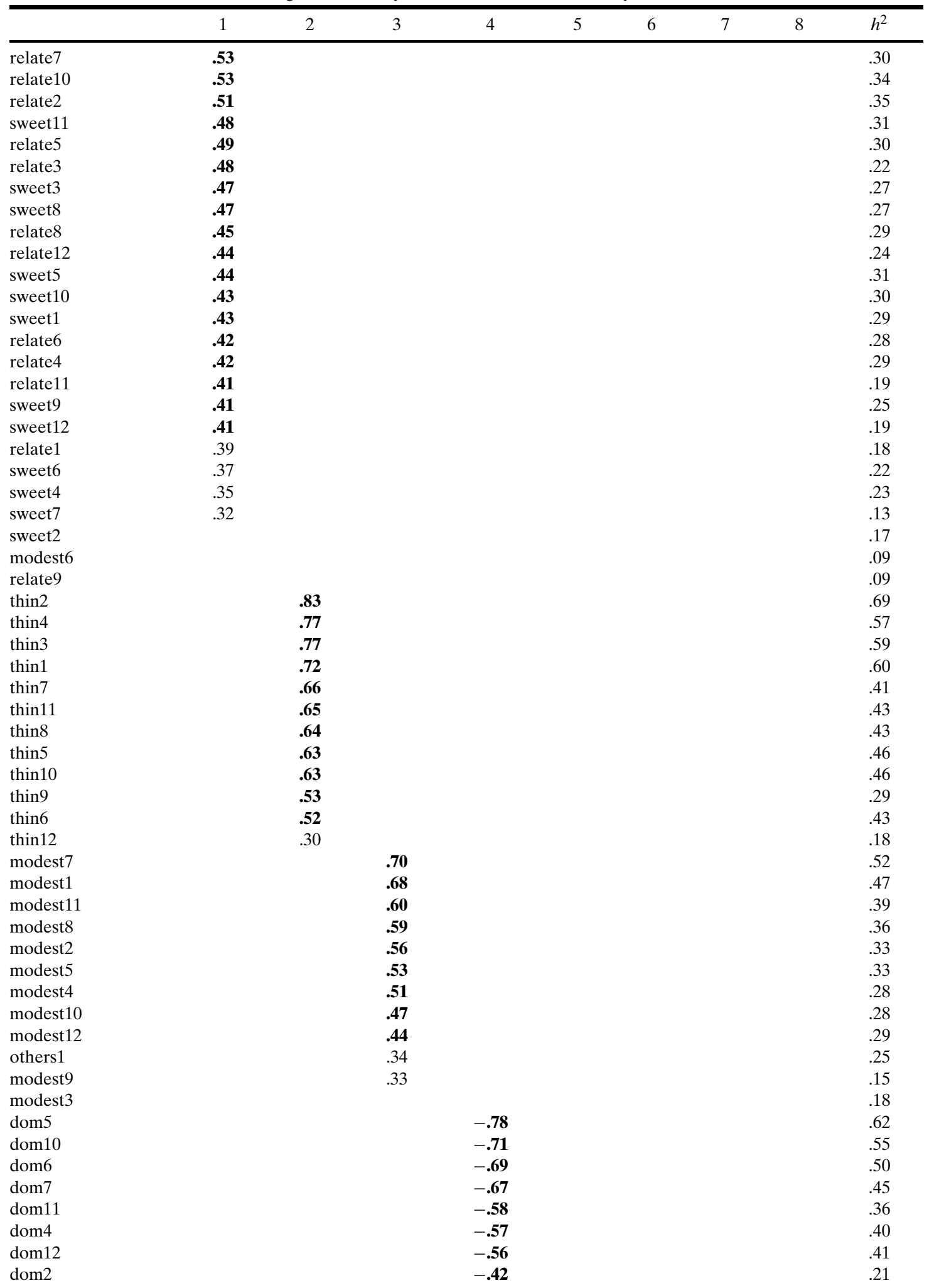


Table I. Continued

\begin{tabular}{|c|c|c|c|c|c|c|c|c|c|}
\hline & 1 & 2 & 3 & 4 & 5 & 6 & 7 & 8 & $h^{2}$ \\
\hline $\operatorname{dom} 3$ & & & & -.39 & & & & & .26 \\
\hline dom1 & & & & -.37 & & & & & .19 \\
\hline $\operatorname{dom} 9$ & & & & -.32 & & & & & .14 \\
\hline $\operatorname{dom} 8$ & & & & & & & & & .06 \\
\hline child4 & & & & & .86 & & & & .73 \\
\hline child9 & & & & & .81 & & & & .61 \\
\hline child3 & & & & & .78 & & & & .60 \\
\hline child12 & & & & & .78 & & & & .63 \\
\hline child10 & & & & & .74 & & & & .54 \\
\hline child7 & & & & & .73 & & & & .53 \\
\hline child11 & & & & & .66 & & & & .49 \\
\hline child1 & & & & & .66 & & & & .48 \\
\hline child2 & & & & & .66 & & & & .42 \\
\hline child5 & & & & & .65 & & & & .45 \\
\hline child 8 & & & & & .64 & & & & .42 \\
\hline child6 & & & & & .60 & & & & .41 \\
\hline roman3 & & & & & & -.62 & & & .44 \\
\hline roman6 & & & & & & -.60 & & & .42 \\
\hline roman5 & & & & & & -.52 & & & .36 \\
\hline roman 9 & & & & & & -.48 & & & .25 \\
\hline roman7 & & & & & & -.45 & & & .24 \\
\hline roman11 & & & & & & -.45 & & & .26 \\
\hline roman1 & & & & & & -.43 & & & .26 \\
\hline roman2 & & & & & & -.43 & & & .24 \\
\hline roman4 & & & & & & -.42 & & & .29 \\
\hline roman10 & & & & & & -.37 & & & .22 \\
\hline roman12 & & & & & & & & & .10 \\
\hline roman8 & & & & & & & & & .12 \\
\hline fidel10 & & & & & & & -.84 & & .68 \\
\hline fidel5 & & & & & & & -.82 & & .65 \\
\hline fidel4 & & & & & & & -.73 & & .52 \\
\hline fidel2 & & & & & & & -.63 & & .41 \\
\hline fidel11 & & & & & & & -.63 & & .40 \\
\hline fidel12 & & & & & & & -.62 & & .37 \\
\hline fidel1 & & & & & & & -.59 & & .38 \\
\hline fidel6 & & & & & & & -.51 & & .33 \\
\hline fidel8 & & & & & & & -.44 & & .24 \\
\hline fidel7 & & & & & & & -.44 & & .25 \\
\hline fidel9 & & & & & & & -.39 & & .25 \\
\hline appear9 & & & & & & & & & .15 \\
\hline fidel3 & & & & & & & & & .11 \\
\hline appear5 & & & & & & & & .76 & .59 \\
\hline appear11 & & & & & & & & .75 & .57 \\
\hline appear10 & & & & & & & & .63 & .46 \\
\hline appear8 & & & & & & & & .60 & .38 \\
\hline appear2 & & & & & & & & .58 & .37 \\
\hline appear7 & & & & & & & & .55 & .34 \\
\hline appear6 & & & & & & & & .40 & .37 \\
\hline appear12 & & & & & & & & .36 & .23 \\
\hline appear1 & & & & & & & & .34 & .39 \\
\hline appear4 & & & & & & & & .33 & .30 \\
\hline appear3 & & & & & & & & & .34 \\
\hline Eigenvalue & 10.64 & 8.82 & 5.43 & 4.93 & 3.90 & 3.30 & 3.11 & 2.81 & \\
\hline$\%$ variance & 9.76 & 8.10 & 4.99 & 4.52 & 3.58 & 3.02 & 2.85 & 2.58 & \\
\hline
\end{tabular}

Note. $N=733 . h^{2}=$ communality of each item. relate $=$ Relational; sweet $=$ Sweet and Nice; thin $=$ Thinness; modest $=$ Modest dom $=$ Be Domestic child $=$ Caring for Children; roman $=$ Romantic Relationship; fidel $=$ Sexual Fidelity; appear = Invest in Appearance. Bolded item values indicate that these items were retained to comprise the subscale for that factor in all subsequent analyses. 
Table II. Definitions and Sample Items for Each of the Eight Subscales of the Conformity to Feminine Norms Inventory

\begin{tabular}{|c|c|c|}
\hline Subscale & Definition of feminine norm & Sample items \\
\hline Nice in relationships & $\begin{array}{l}\text { Develop friendly and supportive } \\
\text { relationships with others }\end{array}$ & $\begin{array}{l}\text { It is important to let people know they are special } \\
\text { I would be ashamed if someone thought I was mean } \\
\text { I don't go out of my way to keep in touch with friends } \\
\text { (reversed) }\end{array}$ \\
\hline Thinness & Pursue a thin body ideal & $\begin{array}{l}\text { I am always trying to lose weight } \\
\text { I would be happier if I was thin } \\
\text { I'd look better if I put on a few pounds (reversed) }\end{array}$ \\
\hline Modesty & $\begin{array}{l}\text { Refrain from calling attention to one's } \\
\text { talents or abilities }\end{array}$ & $\begin{array}{l}\text { I always downplay my achievements } \\
\text { I feel uncomfortable being singled out for praise } \\
\text { There is nothing wrong with bragging (reversed) }\end{array}$ \\
\hline Domestic & Maintain the home & $\begin{array}{l}\text { I do all of the cleaning, cooking, and decorating where } \\
\text { I live } \\
\text { I enjoy spending time making my living space look nice } \\
\text { It is important to keep your living space clean }\end{array}$ \\
\hline Care for children & Take care and be with children & $\begin{array}{l}\text { I would baby-sit for fun } \\
\text { Most people enjoy children more than I do (reversed) } \\
\text { Taking care of children is extremely fulfilling }\end{array}$ \\
\hline Romantic relationship & Invest self in romantic relationship & $\begin{array}{l}\text { Whether I'm in one or not, romantic relationships are } \\
\text { often on my mind } \\
\text { I pity people who are single } \\
\text { I can be happy without being in a romantic relationship } \\
\text { (reversed) }\end{array}$ \\
\hline Sexual fidelity & $\begin{array}{l}\text { Keep sexual intimacy contained within } \\
\text { one committed relationship }\end{array}$ & $\begin{array}{l}\text { I would only have sex if I was in a committed } \\
\text { relationship like marriage } \\
\text { I would feel guilty if I had a one-night stand } \\
\text { There is no greater insult than to be called a "slut" }\end{array}$ \\
\hline Invest in appearance & $\begin{array}{l}\text { Commit resources to maintaining and } \\
\text { improving physical appearance }\end{array}$ & $\begin{array}{l}\text { I never wear make-up (reversed) } \\
\text { I'd feel superficial if I wore make-up (reversed) } \\
\text { It is important to look physically attractive in public }\end{array}$ \\
\hline
\end{tabular}

feelings, and thoughts consistent with the norms that women (1) develop friendly and supportive relationships with others (Nice in Relationships), (2) pursue a thin body ideal (Thinness), (3) refrain from calling attention to one's talents or abilities (Modesty), (4) maintain the home (Domestic), (5) take care and be with children (Care for Children), (6) invest self in romantic relationship (Romantic Relationship), (7) keep sexual intimacy contained within one committed relationship (Sexual Fidelity), and (8) commit resources to maintaining and improving physical appearance (Invest in Appearance). See Table II for sample items for each subscale.

That eight factors were identified also lends support for the idea that there are multiple feminine norms to which women respond and that these norms are distinguishable from each other. Related to this point, we underscore two issues concerning the feminine norms identified in the study. First, we recognize that the final factor structure and the subscales retained through the process were likely affected by the characteristics of the sample. For example, it may be that the Look Young items would have loaded as a distinct factor if the sample were not comprised mostly of college undergraduates. Like all measure development efforts that employ factor analysis, we recognize that our factor structure solution may be sample-dependent, and we look to future research to cross-validate the structure we identified in this study.

Second, the results of the study should in no way suggest that there are only eight feminine normsno more and no less - in the dominant culture in the United States. There are obviously other feminine norms in the dominant culture in the U.S. that our measure development process did not capture. We also recognize that there are other feminine norms from other cultural groups in the U.S., as well as other feminine norms from other cultural groups around the world, that we did not identify. However, we believe that having a measurement tool that allows examination of a larger number of feminine 
norms than the current femininity indices has benefits to research in psychology, and other disciplines, over previous measures. Such a tool allows us to examine the great variability between women, and within individual women in different contexts or longitudinally, in how women enact femininity. Such a measure may also allow a better examination of the resultant benefits and costs of both conformity and nonconformity to feminine norms for the individual and others.

\section{STUDY 2}

The purposes of this study were to examine reliability and validity indicators for the CFNI Total score and the eight Femininity Norms. Specifically, our purposes were (1) to examine the internal consistency estimates for the CFNI Total score and eight Femininity Norms subscales, (2) to examine the intercorrelations among the eight Femininity Norms subscales and item-total correlations of the Femininity Norms with the Total score, (3) to determine whether women score higher on the CFNI scores than men do, and (4) to determine if CFNI scores relate in predicted ways to constructs such as global femininity, global masculinity, and feminist identity development.

The rationale for the third purpose was based on the assumption that women would be more likely to conform to feminine norms than men would. The rationale for the last purpose was based on the assumption that scales on an inventory of conformity to traditional feminine norms would relate positively to an index of global femininity and negatively to an index of global masculinity. As socially desirable gender role characteristics as measured by the BSRI are thought to be related to, but distinct from, gender role ideologies (Thompson \& Pleck, 1995), we anticipated that the relationships between global femininity and the CFNI would be significant but would not have strong correlations.

In addition, because the CFNI is intended to measure conformity to traditional norms of femininity in the U.S., we thought it should also relate to women's development of a feminist identity. In describing women's feminist identity development, Downing and Roush (1985) proposed a five-stage model in which the first stage, passive acceptance, reflects acceptance of traditional European American, North American, gender roles, beliefs that men are superior to women, and that these roles are ad- vantageous. The second stage, revelation, is in response to a crisis or crises that lead women to question traditional gender roles and to have concomitant feelings of anger toward men. Sometimes women in this stage also feel guilty because of how they may have contributed to their own and other women's oppression in the past. The third stage, embeddednessemanation, reflects feelings of connection to other women, cautious interactions with men, and development of a more relativistic frame of life. The fourth stage, synthesis, is when women develop a positive feminist identity and are able to transcend traditional gender roles. The final stage, active commitment, reflects beliefs that men are equal to women but not the same, and reflects a strong commitment to positive social change that addresses societal inequities (see Bargad \& Hyde, 1991; Downing \& Roush, 1985; Fischer et al., 2000; Moradi, Subich, \& Phillips, 2002). As such, we posit that CFNI scores, which were designed to measure conformity to traditional gender role norms in the dominant culture of U.S. society, should be positively related to the passive acceptance stage scores that reflect acceptance of traditional European American, North American, gender roles, but negatively to the other four feminist identity stages as these reflect ever increasing levels of feminist identity.

\section{METHOD}

\section{Participants}

The 733 women described in Study 1 and 98 men participated in this study. Two hundred and eleven of the 733 women also completed additional measures for this study (i.e., the Bem Sex Role Inventory and the Feminist Identity Development Scale), as well as a demographics form and the CFNI described in Study 1.

These 211 women averaged 18.83 years of age $(S D=.73)$ and most were European American (168 European American, 6 African American, 21 Asian or Asian American, 9 Hispanic/Latina, 1 Native American, and 6 biracial). Most women reported that they were single ( 209 single, 1 married, and 1 person did not answer) and heterosexual (208 heterosexual, 2 lesbian, and 1 bisexual).

Men in the study averaged 18.81 years of age $(S D=1.02)$, and most were European American (81 European American, 3 African American, 6 Asian or Asian American, 6 Hispanic/Latino, and 2 biracial). 
All of the men reported that they were single, and almost all identified as heterosexual (96 heterosexual, 1 homosexual, and 1 bisexual).

\section{Measures}

The Bem Sex Role Inventory (BSRI; Bem, 1974, 1981b) assesses femininity and masculinity with 60 -items on which participants are asked to rate themselves using a 7-point scale on 20 femininetyped adjectives, 20 masculine-typed adjectives, and 20 neutral adjectives. Scores of the gender-typed items are averaged to obtain a femininity raw score and a masculinity raw score; higher scores indicate more feminine or masculine traits, respectively. Evidence for the validity of the scale includes its significant relationship to gender-related behavior (Payne, 1985). Further evidence of the validity of the BSRI has been reported in the Bem Sex-Role Inventory Professional Manual (Bem, 1981b) and in Spence (1984). In terms of reliability, Bem (1981b) reported internal consistency estimates that range between .75 and .87 for the Femininity and Masculinity scales, and 4-week test-retest correlations for the two scales that range between .76 and .94 for both genders. In the current study, alpha was .81 for the Femininity score and .86 for the Masculinity score.

The Feminist Identity Composite (FIC; Fischer et al., 2000) was developed to assess feminist identity development as a composite of 33 items from two previous measures of feminist identity [i.e., 20 items from the Feminist Identity Scale (Rickard, 1989) and 13 items from the Feminist Identity Development Scale (Bargad \& Hyde, 1991)]. Fischer et al. (2000) reported a 5-factor structure that resembled Downing and Roush's (1985) model and was reported to predict ego identity status, percep- tions of sexist events, and involvement in women's organizations. Fischer et al. (2000) reported internal consistency estimates that range from .68 to .84 for their first sample and .71 to .86 for their second sample. Moradi and Subich (2002) reported internal consistency estimates that range between .73 to .84 in their study. In the present study, alphas were .74 for Passive Acceptance, .81 for Revelation, .89 for Embeddedness-Emanation, .78 for Synthesis, and .83 for Active Commitment.

\section{Procedure}

The 211 women and 98 men were recruited from a large undergraduate Introduction to Biology class at a private northeastern university. Data were collected via a web-based survey with page encryption and other security procedures. The measures described above, along with informed consent and debriefing pages, were converted to a series of web pages, and participants were provided with an internet address (URL) to complete the survey at a time and place of their convenience. Participants received extra credit from their instructor for completing the survey.

\section{Results}

\section{Internal Consistency}

Examination of the internal consistency estimates on the CFNI for women in the study indicated that coefficient $\alpha$ was .88 for the CFNI Total score. For the Femininity Norm subscales, alphas ranged from .77 for Romantic Relationship to .92 for Caring for Children (see Table III).

Table III. Internal Consistencies and Intercorrelations for Women's Scores on the Total Score and Eight Femininity Norms Subscales of Conformity to Feminine Norms Inventory

\begin{tabular}{|c|c|c|c|c|c|c|c|c|c|}
\hline & 1. & 2. & 3. & 4. & 5. & 6. & 7. & 8. & $\alpha$ \\
\hline 1. Nice in relationships & & & & & & & & & .84 \\
\hline 2. Thinness & .05 & & & & & & & & .90 \\
\hline 3. Modesty & $.08^{*}$ & .03 & & & & & & & .82 \\
\hline 4. Domestic & $.09^{*}$ & $.14^{* * *}$ & $-.09^{*}$ & & & & & & .84 \\
\hline 5. Care for children & $.41^{* * *}$ & -.04 & .05 & $.15^{* * *}$ & & & & & .92 \\
\hline 6. Romantic relationship & $.13^{* *}$ & $.24^{* * *}$ & $-.12^{* *}$ & $.17^{* * *}$ & $.13^{* *}$ & & & & .77 \\
\hline 7. Sexual fidelity & $.18^{* * *}$ & -.06 & $.14^{* * *}$ & $.19^{* * *}$ & $.18^{* * *}$ & $.09^{*}$ & & & .85 \\
\hline 8. Invest in appearance & .05 & $.31^{* * *}$ & $-.17^{* * *}$ & $.17^{* * *}$ & -.01 & $.20^{* * *}$ & $-.08^{*}$ & & .82 \\
\hline 9. Total conformity & $.33^{* * *}$ & $.15^{* * *}$ & .01 & $.25^{* * *}$ & $.28^{* * *}$ & $.27^{* * *}$ & $.18^{* * *}$ & $.15^{* * *}$ & .88 \\
\hline
\end{tabular}

Note. $N=733$. Subscale/total conformity correlations are corrected. ${ }^{*} p<.05 .{ }^{* *} p<.01 .{ }^{* * *} p<.001$. 
Table IV. Means, Standard Deviations and $t$ Values Comparing Women and Men on CFNI Scores

\begin{tabular}{|c|c|c|c|c|c|}
\hline \multirow[b]{2}{*}{ CFNI score } & \multicolumn{2}{|c|}{ Women } & \multicolumn{2}{|c|}{ Men } & \multirow[b]{2}{*}{$t$} \\
\hline & $M$ & $S D$ & $M$ & $S D$ & \\
\hline Nice in relationships & 39.48 & 5.97 & 36.32 & 6.24 & $4.89^{* * *}$ \\
\hline Thinness & 20.87 & 6.18 & 15.33 & 5.32 & $8.48^{* * *}$ \\
\hline Modesty & 13.02 & 3.67 & 12.51 & 3.68 & 1.28 \\
\hline Domestic & 14.10 & 3.10 & 13.86 & 3.97 & $2.67^{* *}$ \\
\hline Care for children & 26.51 & 6.24 & 23.77 & 5.11 & $4.17^{* * *}$ \\
\hline Romantic relationship & 15.64 & 3.73 & 15.60 & 3.63 & .11 \\
\hline Sexual fidelity & 20.22 & 5.55 & 16.91 & 5.98 & $5.50^{* * *}$ \\
\hline Invest in appearance & 12.01 & 3.75 & 6.30 & 2.72 & $14.57^{* * *}$ \\
\hline CFNI Total score & 162.73 & 18.26 & 140.57 & 15.77 & $11.45^{* * *}$ \\
\hline
\end{tabular}

Note. $N$ for the total sample $=831$ ( $n=733$ for women, $n=98$ for men $) ; d f=829$ for all $t$-tests.

${ }^{*} p<.051$-tail. ${ }^{* *} p<.011$-tail. ${ }^{* *} p<.0011$-tail.

\section{CFNI Intercorrelations}

Pearson correlations conducted on the relationships of the eight Femininity Norm subscales to each other and to the Total score on the CFNI are presented in Table III. Results indicate that the subscale scores correlate from moderate to low levels with the corrected CFNI Total score (i.e., CFNI Total score after subtracting that subscale from the Total) and that the subscales correlate with each other in the expected directions. The strength of relationship among the subscales with each other ranges from very low and nonsignificant to a correlation of .41 between Nice in Relationships and Care for Children.

\section{Comparisons of Women and Men on CFNI Scores}

To determine whether women and men differed on conformity to femininity norms, their CFNI Total scores and the eight Femininity Norm scores were compared using one-tailed $t$-tests (see Table IV for means and standard deviations of women and men participants on the Total score and eight Femininity Norms subscale scores). Results indicate that women scored significantly higher than men on the CFNI Total score, as well as on 6 of the 8 Feminine Norms scores (i.e., Nice in Relationships, Sexual Fidelity, Be Domestic, Invest in Appearance, Caring For Children, Thinness). Women did not score significantly higher than men on Modesty and Romantic Relationship.

\section{Relation of CFNI Scores to the Bem Sex Role Inventory and the Feminist Identity Development Scale}

To examine the CFNI Total and the eight Femininity Norm subscales in relation to the Bem Sex Role Inventory, Pearson correlations were computed (see Table V). Results of the analyses indicate that

Table V. Means, Standard Deviations, and Correlations Between CFNI Total and Subscale Scores and Bem Sex Role Inventory and Feminist Identity Composite Subscale Scores

\begin{tabular}{lccccccrr}
\hline & BSRIfem & BSRImas & FICPas & FICRev & FICem & FICSyn & FICAct & Mean $(S D)$ \\
\hline Nice in relationships & $.41^{* * *}$ & -.14 & .05 & .00 & .11 & .13 & .12 & $39.63(5.20)$ \\
Thinness & -.03 & .06 & .10 & -.04 & -.06 & -.07 & -.04 & $21.52(6.39)$ \\
Modesty & .01 & $-.19^{* *}$ & -.02 & -.02 & $-.18^{*}$ & $-.25^{* * *}$ & -.09 & $13.26(3.88)$ \\
Domestic & .05 & -.04 & $.25^{* * *}$ & -.04 & -.05 & -.06 & -.06 & $15.19(3.69)$ \\
Care For children & $.37^{* * *}$ & -.11 & $.15^{*}$ & -.13 & .04 & .08 & .08 & $26.50(5.22)$ \\
Be in a romantic relationship & $.20^{* *}$ & -.13 & $.19^{* *}$ & .04 & .05 & -.11 & -.10 & $15.54(3.29)$ \\
Sexual fidelity & $.30^{* * *}$ & $-.24^{* *}$ & $.14^{*}$ & .05 & -.05 & -.03 & .01 & $22.32(5.61)$ \\
Invest in appearance & .07 & .05 & .10 & -.05 & .04 & .13 & $-.14^{*}$ & $12.28(3.64)$ \\
CFNI total score & $.40^{* * *}$ & $-.20^{* *}$ & $.26^{* * *}$ & -.06 & -.03 & -.03 & -.03 & $166.25(16.81)$ \\
Mean $(S D)$ & $5.19(.56)$ & $4.99(.66)$ & $18.97(4.32)$ & $19.99(4.95)$ & $12.62(3.29)$ & $21.09(2.27)$ & $31.57(4.75)$ & \\
\hline
\end{tabular}

Note. $N=208$. BSRIfem = Bem Sex Role Inventory Feminist Identity; BSRImas = Bem Sex Role Inventory Masculine identity; FICPas $=$ Feminist Identity Scale Passive Acceptance; FICRev = Feminist Identity Scale Revelation; FICEm = Feminist Identity Scale Embeddedness-Emanation; FICSyn = Feminist Identity Scale synthesis subscale; FICAct = Feminist Identity Scale active commitment. ${ }^{*} p<.05$ (2-tail). ${ }^{* *} p<.01$ (2-tail). ${ }^{* * *} p<.001$ (2-tail). 
the CFNI Total scores relate significantly and positively to the BSRI Femininity score, significantly and negatively to the BSRI Masculinity score, and significantly and positively to the FIC Passive Acceptance subscale.

Examination of the Femininity Norms subscales in relation to the BSRI and FIC indicated that Nice In Relationship scores relate to BSRI Femininity, but were unrelated to the BSRI Masculinity or the FIC subscales; Modesty was negatively related to BSRI Masculinity and the Embeddedness-Emanation and Synthesis subscales of the FIC; Domestic was positively related to Passive Acceptance on the FIC; Care for Children scores were positively related to BSRI Femininity and Passive Acceptance on the FIC; Romantic Relationship was positively related to BSRI Femininity and Passive Acceptance on the FIC; Sexual Fidelity was positively related to BSRI Femininity and Passive Acceptance on the FIC, but negatively related to BSRI Masculinity; and Invest in Appearance was negatively related to Active Commitment on the FIC.

\section{Discussion}

The results suggest that the CFNI Total and the eight Femininity Norm subscales are internally consistent, have low to moderate relationships among the subscales, and many of the CFNI subscales differentiate men from women and are related to femininity, masculinity, and feminist identity in the predicted ways. Specifically, there were differences supporting our hypothesis between men and women on the CFNI total score and six of the eight Femininity Norms. We did not find, however, significant differences between men and women on Modesty and Romantic Relationship. As separate sociocultural schemas exist for femininity and masculinity (Bem, 1981a), the fact that these two subscales did not differentiate men from women in this study might suggest that refraining from calling attention to one's talents or abilities and investing oneself in a romantic relationship may not be distinctly feminine norms in the U.S. However, other results from this study support our contention that Modesty and Romantic Relationship are traditional feminine norms from the dominant culture in the U.S. Specifically, Modesty was related significantly and negatively to masculinity, as well as negatively related to the higher levels of feminist identity (i.e., embeddedness-emanation and synthesis), and Romantic Relationship was positively associated with femininity and passive acceptance of traditional, North American, gender roles. Thus, these two scores have significant relationships to the BSRI and FIC constructs in ways that support their validity as indicators of traditional femininity.

Results of our examination of the relationship of the CFNI to the Bem Sex Role Inventory also indicate that the CFNI Total score was related positively to femininity and negatively to masculinity. However, we note that the relationship between the CFNI Total score and the Femininity score was moderate, as predicted. This supports our prediction that socially desirable feminine characteristics are related to, but distinct from, feminine ideologies. Also, we note that the BSRI Femininity score only significantly related to Nice in Relationships, Care for Children, Romantic Relationship, and Sexual Fidelity. We interpret this finding as supporting our contention that the BSRI does not incorporate many salient femininity norms found in U.S. society. These relationships also support Spence and Helreich's (1980) conclusion that the BSRI assesses expressive traits as the BSRI was related to the CFNI scores that reflect caretaking and involvement in relationships.

The results also support our hypothesis that CFNI scores, which were designed to measure conformity to traditional gender role norms in the dominant culture of U.S. society, should be positively related to acceptance of traditional European American, North American, gender roles (i.e., passive acceptance), but negatively to the stages that reflect increasing levels of feminist identity. Although none of the CFNI scores related to the revelation stage, it appears that the CFNI Total score-and the Domestic, Care for Children, Romantic Relationship, and Sexual Fidelity scores-are all associated with believing that traditional European American, North American gender roles are advantageous. Specifically, the CFNI subscales that assess conformity to traditional norms associated with homemaking, childcare, and being married (i.e., faithful in a romantic relationship) were the variables related to passive acceptance of traditional gender roles.

Consideration of the CFNI's relationship to the other stages of feminist identity revealed that the CFNI was unrelated to many of the other stages of feminist identity. However, two of the subscales did correlate with the stages that reflect greater feminist identity. Specifically, Modesty was related negatively to feeling connected to other women (Embededness-Emanation) and developing 
a positive feminist identity (Synthesis). Also, Invest in Appearance related negatively to women's self-report of a strong commitment to positive social change that addresses societal inequities (Active Commitment). As such, women who were more likely to speak up about their accomplishments (i.e., low on Modesty) and less likely to focus on their physical appearance (i.e., low on Invest in Appearance) were more likely to report higher levels of feminist identity.

\section{STUDY 3}

The purpose of this study was to examine the relationship between CFNI scores and scores on the Eating Disorder Inventory (EDI-2; Garner, 1991). Among all of the identified norms of femininity, those involving appearance may be some of the most salient in the lives of women in the U.S. (Crawford \& Unger, 2000). Western culture's stereotype of femininity seems to involve the belief that women should be focused upon both taking care of their own appearance and being thin (Worell \& Todd, 1996). Women who fail to meet these cultural prescriptions for attractiveness may experience sanctions for being "insufficiently feminine" (Fiske \& Stevens, 1993), and internalization of this "thin ideal" has been associated with eating and weight concerns (Low et al., 2003).

A significant body of literature supports the hypothesis that women experience much higher levels of body dissatisfaction than do men. Concerns about physical attractiveness have been found to be more prevalent in girls and women than in boys and men (Mintz \& Betz, 1986; Worell \& Todd, 1996). Demarest and Langer (1996) found that overweight women reported the highest levels of body dissatisfaction in a comparison of body perception among underweight, average-weight, and overweight men and women. Women of average weight in the study reported approximately the same level of body dissatisfaction as did overweight men. Furthermore, these gender differences in levels of body dissatisfaction begin to appear with the onset of adolescence, and they continue well past middle adulthood (Tiggeman \& Pennington, 1996).

The cultural norms for physical attractiveness and the "thin ideal" may be particularly salient for European American women (Crawford \& Unger, 2000). In a study by Powell and Kahn (1995), European American women chose a significantly thinner ideal body size and expressed more concern with weight and dieting than did African American women. Results of that study suggest that European American women experience greater social pressure to be thin than do African American women, which may lead to the higher rates of eating disorders among European American women than among African American women.

It was therefore expected that rigid internalization of feminine norms for appearance (Be Thin and Invest in Appearance), as evidenced by high levels of conformity to these norms, would be associated with higher levels of eating disordered behavior, negative body image, and preoccupation with weight as assessed by the EDI. However, the relationships between other dimensions of femininity besides those that are directly related to appearance may also be associated with eating disordered behaviors. Prior researchers who have attempted to link femininity and eating disordered behaviors have been considerably limited by instruments that tap only limited aspects of gender, typically self-reported personality traits. According to Worell and Todd (1996), "in the face of the broad reach of gender stereotypes and roles, simple measures of trait femininity are not likely to bear the weight of explaining the complex behavior patterns involved in developmental concerns such as depression or disordered eating" (p. 139). Use of the CFNI to assess multiple components of conformity to a broad range of feminine norms may provide a more complex perspective examining the relationship between femininity and eating disordered behavior.

\section{Method}

\section{Participants and Procedure}

Three hundred and eighty women participated in this study. These women were participants in Study 1, but they also completed the Eating Disorder Inventory -2 , along with a demographics form and CFNI described in Study 1 (alphas in this study for the eight Femininity Norms subscales ranged from .77 to .93 , with $\alpha=.89$ for the Total score of the CFNI). These women averaged 19.28 years $(S D=$ 1.27), and most were European American (307 European American, 12 African American, 28 Asian or Asian American, 16 Hispanic/Latina, 1 Native American, 8 biracial, and 8 described themselves as "Other") and heterosexual (369 heterosexual, 1 lesbian, 8 bisexual, and 2 who identified as "Other"). 
Most participants were single (330 single, 45 single and cohabitating, 3 married, and 2 did not report). This group consisted of 163 freshman, 150 sophomores, 40 juniors, and 27 seniors.

\section{Measures}

The Eating Disorder Inventory - 2 (EDI-2; Garner, 1991) is a 64-item self-report measure of symptoms commonly associated with anorexia and bulimia nervosa. Respondents are asked to indicate whether each item applies to them on a 6-point scale that includes always, usually, often, sometimes, rarely, and never. Responses are then recoded from the 6point scale into a 4-point scale that ranges from 0 to 3 , in which 0 is assigned to the three responses that represent the least symptomatic answers, and 3 is assigned to the most symptomatic answer (Garner, 1991).

The EDI-2 was developed to assess psychological characteristics associated with eating disorders based on the assumption that disordered eating is multidimensional in nature (Garner, Olmsted, \& Polivy, 1983). The original factor analysis revealed eight scales: (a) Drive for Thinness, (b) Bulimia, (c) Body Dissatisfaction, (d) Ineffectiveness, (e) Perfectionism, (f) Interpersonal Distrust, (g) Interoceptive Awareness, and (h) Maturity Fears.

The EDI has been used in over 400 studies (Garner, 1991), which support the 8-factor structure (Garner et al., 1983). EDI scores have been reported to discriminate between eating disordered and nonclinical samples (Garner, 1991) and to classify correctly $85 \%$ of participants into bulimic and restrictor subtypes. In addition, the EDI and its subscales have been reported to relate significantly to the Eating Attitudes Test and body size estimations (see Garner, 1991). Internal consistency data reported for the EDI indicate Cronbach alphas that ranged from .82 to .90 (Eberly \& Eberly, 1985). Test-retest reliability correlation coefficients for each of the scales, except Maturity Fears, are reportedly .80 or higher over a 3-week period and range from .47 to .76 across a 1-year period (Crowther, Lilly, Crawford, \& Shepherd, 1992). In the present study, alphas were .77 for Drive for Thinness, .63 for Bulimia, .82 for Body Dissatisfaction, .79 for Ineffectiveness, .35 for Perfectionism, .68 for Interpersonal Distrust, .72 for Interoceptive Awareness, .71 for Maturity Fears, and .91 for the EDI Total score. Because the internal consistency for the Perfectionism scale was so low, it was dropped from further analyses in this study.

\section{Results}

Pearson correlations were computed to examine the CFNI Total and eight Femininity Norm subscales in relation to the Eating Disorder Inventory - 2 (see Table VI). Results from the analyses indicated that the CFNI Total scores related significantly and positively the EDI Total score, as well as to the EDI-2 subscale scores of Drive For Thinness, Bulimia, Body Dissatisfaction, and Interoceptive Awareness.

Examination of the CFNI subscales in relation to the EDI-2 indicated that Nice in Relationships scores were negatively related to Ineffectiveness and Interpersonal Distrust. Thinness scores were significantly and positively related to all of the EDI scores and the Total score. Modesty scores were positively related to the EDI Total score and the subscales of

Table VI. Means, Standard Deviations, and Correlations Between CFNI Total and Subscale Scores and Eating Disorders Inventory -2

\begin{tabular}{lccccccccc}
\hline & Drivethin & Bulim & Bodydiss & Ineffect & Intdis & Inaware & Matufear & EDI Total & Mean $(S D)$ \\
\hline Nice in relationships & -.02 & .02 & -.01 & $-.17^{* *}$ & $-.17^{* *}$ & .02 & -.05 & -.07 & $39.41(6.38)$ \\
Thinness & $.66^{* * *}$ & $.41^{* * *}$ & $.63^{* * *}$ & $.23^{* * *}$ & $.11^{*}$ & $.26^{* * *}$ & $.13^{*}$ & $.56^{* * *}$ & $20.74(6.17)$ \\
Modesty & .05 & .06 & .07 & $.17^{* *}$ & $.24^{* *}$ & $.12^{*}$ & .06 & $.15^{* *}$ & $12.79(3.56)$ \\
Domestic & .07 & -.01 & -.03 & -.07 & -.07 & -.06 & -.09 & -.04 & $14.73(4.07)$ \\
Care for children & -.06 & -.08 & -.03 & $-.20^{* * *}$ & $-.18^{* *}$ & -.10 & -.09 & $-.15^{* *}$ & $25.87(6.45)$ \\
Be in a romantic & $.13^{*}$ & $.16^{* *}$ & $.11^{*}$ & $.16^{* *}$ & -.08 & $.19^{* * *}$ & .09 & $.18^{* * *}$ & $15.35(3.81)$ \\
$\quad$ relationship & & & & & & & & \\
Sexual fidelity & -.05 & -.10 & .02 & -.09 & -.06 & -.02 & -.05 & -.06 & $21.62(5.95)$ \\
Invest in appearance & $.25^{* * *}$ & $.14^{* *}$ & $.18^{* * *}$ & .09 & .07 & $.11^{*}$ & .02 & $.20^{* * *}$ & $11.87(3.81)$ \\
CFNI total score & $.27^{* * *}$ & $.15^{* *}$ & $.26^{* * *}$ & -.01 & -.07 & $.12^{*}$ & -.01 & $.18^{* * *}$ & $162.39(19.15)$ \\
Mean $(S D)$ & $6.11(4.91)$ & $3.19(3.71)$ & $9.87(6.00)$ & $3.89(4.88)$ & $2.58(3.02)$ & $5.88(5.61)$ & $5.48(4.50)$ & $44.17(22.27)$ &
\end{tabular}

Note. $N=380$. Drivethin = Drive for Thinness; Bulim = Bulimia; Bodydiss = Body Dissatisfaction; Ineffect = Ineffectiveness; Intdis = Interpersonal Distrust; Inaware $=$ Interoceptive Awareness; Matufear = Maturity Fears.

${ }^{*} p<.05$ (2-tail). ${ }^{* *} p<.01$ (2-tail). ${ }^{* * *} p<.001$. 
Ineffectiveness, Interpersonal Distrust, and Interoceptive Awareness. Care For Children scores were negatively related to the EDI Total score and the subscales Ineffectiveness and Interpersonal Distrust. Romantic Relationship scores were positively related to the EDI Total score as well as the subscales of Drive For Thinness, Bulimia, Body Dissatisfaction, Ineffectiveness, and Interoceptive Awareness. Invest in Appearance scores were positively related to the EDI Total score and the subscales of Drive For Thinness, Bulimia, Body Dissatisfaction, Perfectionism, and Interoceptive Awareness. The Domestic and Sexual Fidelity scores of the CFNI were unrelated to any EDI scores.

\section{Discussion}

The results of this study present a complex picture of femininity's relationship to women's eating and body image issues. As predicted, there was a significant, positive relationship between the CFNI Total score and the EDI total score, which suggests that conformity to the dominant culture's norms of femininity may be related to the development of eating disordered symptomatology. The positive correlations between the CFNI Total score and the EDI subscale scores, including Drive for Thinness, Bulimia, Body Dissatisfaction, and Interoceptive Awareness also support this conclusion. Similar patterns were found between specific CFNI subscales related to appearance and EDI scores. For example, Thinness was positively associated with all six EDI subscales as well as the EDI Total score, and Invest in Appearance was positively correlated with 5 of the 6 EDI subscales (Drive for Thinness, Bulimia, Body Dissatisfaction, Perfectionism, and Interoceptive Awareness), along with the EDI Total score. As such, the results of this study support the hypothesis that conformity to feminine norms generally, as well as appearance-related norms specifically, are positively associated with eating disorder symptomatology as assessed by the EDI.

However, our results also suggest that conformity to some norms of femininity that are not appearance-related may also be associated with behaviors related to eating disorders. For instance, Modesty was positively associated with EDI total scores, as well as the Ineffectiveness, Interpersonal Distrust, and Interoceptive Awareness subscales. It seems plausible that the forces that lead a woman to feel she must downplay her accomplishments and assets might contribute to her feeling inadequate in a number of domains, including her abilities to manage her life, her relationships, and even her own internal emotional states as reflected in these three EDI subscales. A second CFNI subscale, Romantic Relationship, was positively correlated with the EDI Total score and the following subscale scores: Drive for Thinness, Bulimia, Body Dissatisfaction. Ineffectiveness, and Interoceptive Awareness. We can speculate that women may experience their physical attractiveness (particularly their body shape and size) as playing a crucial role in their ability to establish and maintain a relationship with a male partner. From a sociobiological approach, women's attention to their physical attractiveness ensures that they will be selected as desirable mates. A focus upon appearance may allow women to feel a sense of control in a situation that might otherwise elicit feelings of powerlessness and confusion. It is not surprising, therefore, that women who place great value upon the attainment of romantic relationships may also experience considerable self-scrutiny in terms of their physical appearance, and thus engage in the associated dieting behaviors.

It is interesting that some femininity factors, specifically Nice in Relationships and Care for Children, actually seem to be associated with healthier eating attitudes. The Nice in Relationships subscale was negatively associated with two EDI subscales: Ineffectiveness and Interpersonal Distrust. It seems plausible that women who put a great deal of time, care, and attention into their relationships derive a sense of competence from their abilities to maintain these connections, as well as a sense of stable attachment and trust in these partners as a result of their interpersonal efforts. A similar effect may contribute to the negative associations between the Caring for Children norm and the following EDI scores: the Total score, Ineffectiveness, and Interpersonal Distrust. Women who devote their time and energy to nurturing and caring for children, or plan to do so, may derive a sense of competence and connection from these interactions, which may detract from the pressure to focus their identity and success on their appearance.

In summary, this study supports the assumption that body dissatisfaction and engagement in weightcontrol behaviors are indeed positively associated with conformity to appearance-related feminine norms. This study also highlights the complexity of the relationship between femininity and eating disorder symptomatology. That is, as expected, 
appearance-related norms of femininity were positively associated with eating and body issues. However, other elements of traditional femininity, including Being in a Romantic Relationship and Modesty, also seem to play a role in the development of these issues, and other feminine norms, including Nice in Relationships and Care for Children, were associated with fewer of these symptoms.

\section{STUDY 4}

This study was designed to examine the temporal stability of the CFNI Total and its eight Femininity Norm subscales, as further indicators of its reliability.

\section{Method}

\section{Participants and Procedure}

Thirty-nine women participated in this part of the study. All of these women also participated in Study 1, but in addition to the data they provided for the factor analysis, these participants also completed the CFNI again 2-3 weeks after the first administration. The 39 women averaged 27.10 years of age $(S D=6.60)$, and most were European American (34 European American, 2 Asian or Asian American, and 3 Hispanic/Latina). Most women reported that they were single ( 34 single, 2 married, 1 divorced, 1 in committed same-sex partner, and 1 committed male partner).

\section{Results}

To examine the temporal stability for a 2-3 week time period, CFNI scores at Time 1 were correlated with CFNI scores at Time 2. Test-retest coefficients (i.e., Pearson correlations) were .94 for the Total CFNI score, and .83 for Nice in Relationships, .93 for Sexual Fidelity, .83 for Be Domestic, .91 for Invest in Appearance, .95 for Caring for Children, .85 for Modest, .86 for Thinness, and .86 for Romantic Relationship.

\section{GENERAL DISCUSSION}

The results from the four studies provide support for the reliability and validity of the CFNI for the mostly European American, heterosexual, and young adult population sampled. Specifically, results from the studies suggest that the CFNI has eight distinct factors, that these factors relate in theoretically consistent ways to the other constructs we examined, which supports its validity, and that the inventory has high internal consistency estimates and test-retest reliabilities over a 2-3 week period.

As such, we view our results as supporting the work of scholars who have identified a large number of normative messages associated with femininity (e.g., see Crawford \& Unger, 2000; Gilbert \& Scher, 1999). Specifically, the CFNI, as a multidimensional measure of femininity, seems able to differentiate among several of the distinct cultural injunctions concerning femininity in the dominant culture in U.S. society today.

\section{Potential Uses for the CFNI}

The CFNI was developed as a tool for use by researchers and clinicians to examine femininity issues with individuals by assessing conformity to an array of femininity norms. In terms of research, the CFNI could be used to examine the complex, multidimensional ways that women experience and respond to femininity issues in their lives. Researchers might also examine whether variables such as SES, cultural membership, and the gender role norms of important peer groups-as examples of social influence factors-and variables such as self-esteem or attachment to parents-as examples of individual difference factors-predict femininity in women. It can also be used to test Mahalik et al.'s (2003) supposition that there are benefits and costs to the individual and others for conforming to, and not conforming to, a variety of gender role norms. Testing this could be done in a variety of ways including by examining data on psychological health, identity development, health-related behaviors, quality of relationships, or a host of other variables to determine benefits and costs for the individual and others to conformity or nonconformity to an array of femininity norms.

As mental health professionals have advocated increasingly for integrating a gendered perspective into assessment and treatment with clients (e.g., Brooks \& Good, 2001; Brown, 1986; Gilbert \& Scher, 1999; Good, Gilbert, \& Scher, 1990; Mahalik, 1999a, 1999 b), once normed, the CFNI may provide such a tool for the assessment of a large number of feminine norms with female clients, which would allow a richer understanding of the salient aspects of femininity for 
a given individual. Although research must be done to determine if interventions that incorporate interpretation of the CFNI with clients are helpful and for what issues, consistent with feminist theory (Brown, 1986), we speculate that it may be useful to explore with female clients how their conformity or nonconformity to any of the femininity norms both benefits them in daily living as well as contributes stress to their relationships, work, and health. Clinical interventions might focus on helping female clients to change aspects of the gendered self in which the costs associated with conformity or nonconformity outweigh the benefits the client experiences.

\section{Limitations}

The fact that the samples were mostly European American, heterosexual, college students in the United States is a limitation to both the generalizability of the findings and to our knowledge about the psychometric properties of the CFNI with other groups of women. As femininity is a culturally defined construct (Kimmel, 2000; Mead, 1935) that varies as a function of life-stage (Sheehy, 1976), race, and ethnicity (Vasquez, 2003; Wyche, 2001), and changes through history (Kimmel, 2000), it is important from a psychometric perspective to examine other groups of women to determine if the CFNI has the same 8-factor structure and acceptable psychometric properties with other groups of women.

It is also vitally important from a theoretical and clinical perspective to examine groups of women from diverse backgrounds in future research. Specifically, as Mahalik et al. (2003) stated that gender role norms from the most powerful culture in a society affect the experiences of persons in that group, as well as persons in all other groups, we believe that "minority women" (e.g., racial minorities, sexual orientation minorities) and women from less powerful groups (e.g., lower SES groups) are affected by the dominant culture's notions of femininity.

Further, Lazur and Majors (1995) wrote that, "For a man of color, defining his own gender role involves integration of the dominant society's restrictions. Measuring himself against the standard that dictates the male gender role for the dominant culture yet denies equal access to the opportunities that sustain that standard evokes in the man of color frustrations, unexpressed emotions, and a drive for survival" (p. 340). We believe that minority women also experience the same dynamics and that use of the
CFNI with minority women would allow the opportunity to explore these issues in research and clinical practice. Questions could be asked, such as "how does racial identity contribute to minority women's identification with or non-identification with feminine norms from the dominant culture?" The same dynamics should also be relevant for issues such as acculturation, sexual identity development, or an array of other sociocultural variables. Because we believe that there are both benefits and costs to conformity and nonconformity to feminine norms in the dominant culture, a measure that assesses specifically the dominant culture's construction of femininity has utility for scientists and practitioners who address minority women's concerns.

In conclusion, we believe that the results of these studies offer initial evidence supporting the reliability and validity of the 8-factor CFNI. Given that mental health professionals are increasingly integrating a gendered perspective into their work, the CFNI may provide a useful tool in working with female clients, as well as a useful tool to examine important questions about the benefits and costs for individuals, families, and communities as a function of conformity and nonconformity to feminine norms.

\section{ACKNOWLEDGMENTS}

We would like to thank all of the women who participated in the first focus groups, the graduate students who participated in the second focus groups, Sam Wan for technical help with the survey, and Mari Casares for her contribution to the group interviews.

\section{REFERENCES}

Bargad, A., \& Hyde, J. S. (1991). A study of feminist identity development in women. Psychology of Women Quarterly, 15, 181-201.

Bartlett, M. S. (1950). Tests of significance in factor analysis. British Journal of Psychology, 3, 77-85.

Bem, S. L. (1974). The measurement of psychological androgyny. Journal of Consulting and Clinical Psychology, 42, 155-162.

Bem, S. L. (1981a). Gender schema theory: A cognitive account of sex typing. Psychological Review, 88, 354-364.

Bem, S. L. (1981b). Bem Sex Role Inventory professional manual. Stanford, CA: Consulting Psychologists.

Betz, N. E. (1995). Gender-related individual differences variables: New concepts, methods, and findings. In D. J. Lubinski, \& R. V. Dawis (Eds.), Assessing individual differences in human behavior: New concepts, methods, and findings (pp. 119143). Palo Alto, CA: Davies-African American.

Brooks, G. R., \& Good, G. E. (Eds.). (2001). The new handbook of psychotherapy and counseling with men: A comprehensive 
guide to settings, problems, and treatment approaches (Vol. 1 \& 2). San Francisco: Jossey-Bass.

Brown, L. S. (1986). Gender role analysis: A neglected component of psychological assessment. Psychotherapy, 23, 243-248.

Brown, L. S. (1998). Raising their voices: The politics of girls' anger. Cambridge, MA: Harvard University Press.

Carpenter, L. M. (2002). Gender and the meaning and experience of virginity loss in the contemporary United States. Gender and Society, 16, 345-365.

Chodorow, N. (1978). The reproduction of mothering. Berkeley, CA: University of California Press.

Cialdini, R. B., \& Trost, M. R. (1999). Social influence: Social norms, conformity, and compliance. In D. Gilbert, S. Fiske, \& G. Lindzy (Eds.), The handbook of social psychology (Vol. 2, pp. 151-192). Boston: McGraw-Hill.

Crawford, M., \& Unger, R. (2000). Women and gender: A feminist psychology. Boston: McGraw-Hill.

Crowther, J. H., Lilly, R. S., Crawford, P. A., \& Shepherd, K. L. (1992). The stability of the Eating Disorder Inventory. International Journal of Eating Disorders, 12, 97-101.

Deaux, K., \& Lewis, L. L. (1984). The structure of gender stereotypes: Interrelatioships among components and gender labels. Journal of Personality and Social Psychology, 46, 991-1004.

Demarest, J., \& Langer, E. (1996). Perception of body shape by underweight, average, and overweight men and women. Perceptual and Motor Skills, 83, 569-570.

Deutsch, F. M., \& Saxon, S. E. (1998). Traditional ideologies, nontraditional lives. Sex Roles, 38, 331-362.

Downing, N., \& Roush, K. (1985). From passive acceptance to active commitment: A model of feminist identity development for women. Counseling Psychologist, 13, 695-709.

Eagly, A. H., \& Steffen, V. J. (1984). Gender stereotypes stem from the distribution of women and men into social roles. Journal of Personality and Social Psychology, 46, 735-754.

Eberly, C. C., \& Eberly, B. W. (1985). A review of the Eating Disorder Inventory. Journal of Counseling and Development, 64, 285.

Eisler, R. M. (1995). The relationship between masculine gender role stress and men's health risk: The validation of the construct. In R. F. Levant \& W. S. Pollack (Eds.), A new psychology of men (pp. 207-225). New York: Basic Books.

Fischer, A. R., Tokar, D. M., Mergl, M. M., Good, G. E., Hill, M. S., \& Blum, S. A. (2000). Assessing women's feminist identity development: Studies of convergent, discriminant, and structural validity. Psychology of Women Quarterly, 24, 1529.

Fiske, S. T., \& Stevens, L. E. (1993). What's so special about sex? Gender stereotyping and discrimination. In S. Oskamp \& M. Costanzo (Eds.), Gender issues in contemporary society: Claremont Symposium on Applied Social Psychology (Vol. 6, pp. 173-196). Thousand Oaks, CA: Sage.

Garner, D. M. (1991). Eating Disorder Inventory - 2 manual. Odessa, FL: Psychological Assessment Resources.

Garner, D. M., Olmsted, M. P., \& Polivy, J. (1983). Development and validation of a multidimensional Eating Disorder Inventory for anorexia and bulimia. International Journal of Eating Disorders, 2, 15-34.

Gilbert, L. A., \& Scher, M. (1999). Gender and sex in counseling and psychotherapy. Needham Heights, MA: Allyn \& Bacon.

Gillespie, B. L., \& Eisler, R. M. (1992). Development of the Feminine Gender Role Stress Scale: A cognitive-behavioral measure of stress, appraisal, and coping for women. Behavior Modification, 16, 426-438.

Gillespie, R. (2003). Childfree and feminine: Understanding the gender identity of voluntarily childless women. Gender and Society, 17, 122-136.

Gilligan, C. (1982). In a different voice: Psychological theory and women's development. Cambridge, MA: Harvard University Press.
Good, G. E., Gilbert, L. A., \& Scher, M. (1990). Gender aware therapy: A synthesis of feminist therapy and knowledge about gender. Journal of Counseling and Development, 68, 376-380.

Green, P. E. (1976). Mathematical tools for applied multivariate analysis. New York: Academic.

Hurd, L. C. (1999). "We're not old!": Older women's negotiation of aging and oldness. Journal of Aging Studies, 13, 419-439.

Jordon, J. V. (1992). The relational self: A new perspective for understanding women's development. Contemporary Psychotherapy Review, 7, 56-71.

Kagan, J. (1964). American longitudinal research on psychological development. Child Development, 35, 1-32.

Kaiser, H. F. (1974). An index of factorial simplicity. Psychometrika, 39, 31-36.

Kimmel, M. S. (2000). The gendered society. Oxford: Oxford University Press.

Kohlberg, L. (1966). Moral education in the schools: A developmental view. School Review, 74, 1-30.

Lazur, R. F., \& Majors, R. (1995). Men of color: Ethnocultural variations of male gender role strain. In R. F. Levant \& W. S. Pollack (Eds.), The new psychology of men (pp. 337-358). New York: Basic Books.

Locksley, A., \& Colten, M. E. (1979). Psychological androgyny: A case of mistaken identity. Journal of Personality and Social Psychology, 37, 1017-1031.

Low, K. G., Charanasomboon, S., Brown, C., Hiltunen, G., Long, K., Reinhalter, K., et al. (2003). Internalization of the thin ideal, weight, and body image concerns. Social Behavior and Personality, 31, 81-90.

Lytton, H., \& Romney, D. M. (1991). Parents' differential socialization of boys and girls: A meta-analysis. Psychological Bulletin, 109, 267-296.

Mahalik, J. R. (1999a). Interpersonal psychotherapy with men who experience gender role conflict. Professional Psychology: Research and Practice, 30, 5-13.

Mahalik, J. R. (1999b). Incorporating a gender role strain perspective in assessing and treating men's cognitive distortions. Professional Psychology: Research and Practice, 30, 333-340.

Mahalik, J. R., Locke, B., Ludlow, L., Diemer, M., Scott, R. P. J., Gottfried, M., et al. (2003). Development of the Conformity to Masculine Norms Inventory. Psychology of Men and Masculinity, 4, 3-25.

Matschiner, M., \& Murnen, S. K. (1999). Hyperfeminity and influence. Psychology of Women Quarterly, 23, 631-642.

Mead, M. (1935). Sex and temperament in three primitive societies. New York: Morrow.

Meth, R. (1990). The road to masculinity. In R. Meth \& R. Pasick (Eds.), Men in therapy: The challenge of change (pp. 3-34). New York: Guilford.

Mintz, L. B., \& Betz, N. E. (1986). Sex differences in the nature, realism, and correlates of body image. Sex Roles, 15, 185-195.

Moradi, B., \& Subich, L. M. (2002). Feminist identity development measures: Comparing the psychometrics of three instruments. Counseling Psychologist, 30, 66-86.

Moradi, B., Subich, L. M., \& Phillips, J. C. (2002). Revisiting feminist identity development theory, research, and practice. Counseling Psychologist, 30, 6-43.

Morawski, J. G. (1985). The measurement of masculinity and femininity: Engendering categorical realities. Journal of Personality, 53, 196-223.

Payne, F. D. (1985). Review of the Bem Sex-Role Inventory. In J. V. Mitchell, Jr. (Ed.), The ninth mental measurements yearbook (pp. 178-179). Lincoln, NE: University of Nebraska Press.

Pleck, J. H. (1981). The myth of masculinity. Cambridge, MA: MIT Press.

Pleck, J. H. (1995). The gender role strain paradigm: An update. In R. F. Levant \& W. S. Pollack (Eds.), A new psychology of men (pp. 11-32). New York: Basic Books. 
Powell, A. D., \& Kahn, A. S. (1995). Racial differences in women's desires to be thin International Journal of Eating Disorders, 17, 191-195.

Rickard, K. M. (1989). The relationship of self-monitored dating behavior to level of feminist identity on the Feminist Identity Scale. Sex Roles, 20, 213-226.

Rudman, L. A., \& Glick, P. (2001). Prescriptive gender stereotypes and backlash toward agentic women. Journal of Social Issues, $57,743-762$.

Sheehy, G. (1976). Passages: Predictable crises of adult life. New York: Bantam.

Spence, J. T. (1984). Masculinity, femininity, and gender-related traits: A conceptual analysis and critique of current research. In B. A. Maher \& W. B. Maher (Eds.), Progress in experiemental research in personality (Vol. 13, pp. 1-97). New York: Academic.

Spence, J. T., \& Helmreich, R. L. (1978). Masculinity and femininity: Their psychological dimensions, correlates and antecedents. Austin, TX: University of Texas Press.

Spence, J. T., \& Helmreich, R. L. (1980). Masculine instrumentality and feminine expressiveness: Their relationships with sex role attitudes and behaviors. Psychology of Women Quarterly, 5, 147-163.

SPSS (1999). SPSS reference guide. Chicago: SPSS, Inc.
Thompson, E. H., \& Pleck, J. H. (1995). Masculine ideologies: A review of research instrumentation on men and masculinities. In R. F. Levant \& W. S. Pollack (Eds.), The new psychology of men (pp. 129-163). New York: Basic Books.

Tiggeman, M., \& Pennington, B. (1996). The development of gender differences in body-size dissatisfaction. Australian Psychologist, 25, 306-313.

Tolman, D. L., \& Porche, M. V. (2000). The Adolescent Feminine Ideology Scale: Development and validation of a new measure for girls. Psychology of Women Quarterly, 24, 365376.

Vasquez, M. J. T. (2003). The Counseling Psychologist: 2002 Presidential Address: Extending the ladder of opportunitybreaking through the colored glass ceiling. Counseling Psychologist, 31, 115-128.

Worell, J., \& Todd, J. (1996). Development of the gendered self. In L. Smolak \& M. P. Levine (Eds.), The developmental psychopathology of eating disorders: Implications for research, prevention, and treatment (pp. 135-156). Hillsdale, NJ: Erlbaum.

Wyche, K. F. (2001). Sociocultural issues in counseling for women of color. In R. K. Unger (Ed.), Handbook of the psychology of women and gender (pp. 330-340). New York: Wiley. 